УДК 616.9:578.826.1]-06:616.34-002

DOI: $10.26435 /$ UC.V0I4(37).618

\author{
А.В. Налетов, Д.В. Каспир, Н.П. Гуз, О.С. Налетова
}

ГОО ВПО «Донецкий национальный медицинский университет имени М. Горького», Донецк

\title{
ОСОБЕННОСТИ КЛИНИЧЕСКИХ ПРОЯВЛЕНИЙ ПОСТИНФЕКЦИОННОГО СИНДРОМА РАЗДРАЖЕННОГО КИШЕЧНИКА У ПАЦИЕНТОВ, ПЕРЕНЕСШИХ COVID-19
}

Эпидемическая ситуация, которая сложилась на сегодняшний день в мире, является серьезным испытанием для каждого региона земного шара, а коронавирусная инфекция ежедневно продолжает забирать жизни тысяч людей, независимо от пола, возраста и этнической принадлежности. В большинстве исследований основное внимание исследователей уделяется рассмотрению клинических симптомов, характерных для поражения легких, определяющих в большинстве случаев прогноз заболевания. Между тем, при COVID-19 могут поражаться и другие системы организма, в том числе и органы желудочно-кишечного тракта [1].

Известно, что коронавирус проникает в организм через рецепторы ангиотензинпревращающего фермента 2 (АПФ2, angiotensin converting enzyme 2, ACE2). Высокая экспрессия рецепторов АПФ2 при COVID-19 отмечается не только в альвеолярных клетках легких, но и в железистых эпителиальных клетках желудка, двенадцатиперстной кишки и прямой кишки, что может приводить к возникновению гастроинтестинальных симптомов у пациентов. Так, согласно результатам ряда исследований, у 10-50\% больных с коронавирусной инфекцией отмечаются гастроэнтерологические жалобы $[2,3]$.

Одним из наиболее распространенных в популяции функциональных гастроинтестинальных расстройств (ФГИР) на сегодняшний день является синдром раздраженного кишечника (СРК). Согласно эпидемиологическим данным, СРК наблюдается у 10-15\% населения планеты, однако лишь 25-30\% из этих больных обращаются за медицинской помощью. Манифестация симптомов заболевания обычно происходит в молодом возрасте - до 30 лет. У женщин СРК развивается чаще, чем у мужчин [4-6].

Основополагающим документом, регламентирующим на сегодняшний день диагностику и лечение ФГИР у детей и взрослых, являет- ся Римский консенсус IV (2016). Согласно данного документа, СРК диагностируют при наличии следующих симптомов: рецидивирующая боль в животе, возникающая по меньшей мере 1 раз в неделю, характеризующаяся следующими признаками (двумя или более): связана с дефекацией; сочетается с изменением частоты дефекаций; сочетается с изменением консистенции кала. Симптомы возникают в течение последних 3 месяцев при общей продолжительности наблюдения не менее 6 месяцев $[4,5]$.

В классификации СРК, в зависимости от характера изменений формы кала, согласно Бристольской шкале формы кала, выделяют: СРК с преобладанием запора, СРК с преобладанием диареи, смешанный вариант СРК, неклассифицируемый вариант СРК [5].

Также в последние годы получила распространение классификация СРК, основанная на превалирующем этиологическом факторе: постинфекционный СРК (ПИ-СРК); вариант расстройства, связанный с непереносимостью пищевых продуктов; классический СРК, индуцированный стрессом [4, 6-8].

\section{ЦЕЛ Ь РАБОТЫ}

Оценить особенности клинического течения ПИ-СРК у пациентов, перенесших COVID-19.

\section{МАТЕРИАЛЫ И МЕТОДЫ}

На базе ООО «Медицинский центр Гастролайн г. Донецка» было обследовано 60 пациентов с СРК. У 30 пациентов был диагностирован ПИ-СРК на фоне перенесенной коронавирусной инфекции (основная группа). У данных больных на основании клинико-лабораторных и рентгенологических результатов обследования был диагностирован COVID-19, проведено соответствующее лечение с клиническим выздоровле-

( А.В. Налетов, Д.В. Каспир, Н.П. Гуз, О.С. Налетова, 2020 (c) Университетская Клиника, 2020 
нием и получением двукратного отрицательного результата при лабораторном исследовании на наличие SARS-CoV-2.

Группу сравнения составили 30 больных с классическим СРК, индуцированным стрессом. Статистически значимой разницы по возрасту и по полу между группами сравнения не выявлено (p>0,05). В обеих группах превалировали лица женского пола - 3:1.

Диагностика СРК основывалась на рекомендациях Римского консенсуса IV (2016). Для диагностики типа СРК была дополнительно использована Бристольская шкала формы кала.

Для оценки наличия и выраженности основных клинических симптомов ФГИР у обследованных пациентов был использован Опросник «7х7» (7 симптомов за 7 дней), разработанный Российской гастроэнтерологической ассоциацией и рекомендованный Резолюцией Экспертного совета, посвященной проблемам диагностики и лечения функциональных заболеваний ЖКТ. В опросник включены вопросы о наличии, выраженности и частоте семи основных симптомов ФГИР за последние 7 дней. В зависимости от полученной суммы баллов тяжесть состояния больного оценивали следующим образом: 0-1 - здоров; 2-6 - пограничное расстройство; 7-12 - легкое расстройство; 13-18 - умеренно выраженное расстройство; 19-24 - выраженное расстройство; 25 и более - тяжелое расстройство [9].

Для оценки степени тяжести абдоминального болевого синдрома была использована простая описательная шкала интенсивности боли, при помощи которой пациент характеризовал наличие абдоминального болевого синдрома: отсутствие боли, легкая, умеренная, сильная, очень сильная, невыносимая боль.

Для проведения анализа результатов исследования использовали методы биостатистики. Для оценки результатов количественных характеристик в работе приводится значение среднего арифметического $(\overline{\boldsymbol{X}})$ оцениваемого параметра и значение ошибки среднего (m). Перед началом анализа выборки проверялись на нормальный закон распределения. В случае нормального закона распределения различия опре- деляли путем сравнения двух средних. Для этого использовался критерий Стьюдента для независимых выборок. Для оценки результатов качественных характеристик приводится значение показателя частоты проявления признака (\%) и его стандартная ошибка (m \%). Для сравнения качественных признаков использовали критерий $\chi^{2}$. Результаты обработки данных в тексте представлены в форме показателя частоты проявления признака (\%) и его 95 \% доверительного интервала (95 \% ДИ).

\section{РЕЗУЛЬТАТЫ И ОБСУЖДЕНИЕ}

Согласно данным проведенного анализа результатов анкетирования с использованием опросника «7х7», установлено, что пациенты основной группы имели более высокие баллы в оценке выраженности клинических симптомов ФГИР. Так, средний балл по опроснику «7х7» среди пациентов основной группы составил $16,3 \pm 0,3$, а среди пациентов группы сравнения $-12,4 \pm 0,2$. Уровень значимости отличий между группами сравнения по данному показателю составил $\mathrm{p}<0,001$.

Как представлено в таблице 1, среди пациентов основной группы преобладала умеренная и выраженная степень тяжести ФГИР - 50,0 \% (95 \% ДИ 31,0 - 69,0 \%) и 40,0 \% (95 \% ДИ 21,4 - 58,6 \%) больных соответственно. Легкая степень тяжести расстройства была установлена статистически значимо $(\mathrm{p}<0,05)$ реже - лишь у 10,0 \% (95 \% ДИ 1,4 - 21,4 \%) больных данной группы. Таким образом, для пациентов с ПИ-СРК, перенесших COVID-19, характерным было наличие умеренной и выраженной степени тяжести расстройства.

В группе сравнения легкая степень тяжести расстройства была установлена у 46,7 \% (95 \% ДИ 27,7 - 65,6 \%) пациентов, а умеренная степень - у 43,3 \% (95 \% ДИ 24,5 - 62,2 \%). При этом доля больных с выраженным расстройством в группе сравнения была статистически значимо (p<0,05) наименьшей - 10,0 \% (95 \% ДИ 2,0 24,0 \%) пациентов. Таким образом, для пациентов, страдающих классическим СРК, характерным было наличие легкой и умеренной степени тяжести ФГИР.

Таблица 1.

Распределение пациентов по степени тяжести ФГИР с использованием опросника «7х7» в группах

\begin{tabular}{lcc}
\hline \multicolumn{1}{c}{ Степень тяжести расстройства } & $\begin{array}{c}\text { Основная груп- } \\
\text { па }(\mathrm{n}=30) \text { абс. }(\% \pm \mathrm{m} \%)\end{array}$ & $\begin{array}{c}\text { Группа сравнения } \\
(\mathrm{n}=30) \text { aбc. }(\% \pm \mathrm{m} \%)\end{array}$ \\
\hline \hline Легкое расстройство & $3(10,0 \pm 5,6 \%)$ & $14(46,7 \pm 9,3 \%)$ \\
\hline Умеренное расстройство & $15(50,0 \pm 9,3 \%)$ & $13(43,3 \pm 9,2 \%)$ \\
\hline Выраженное расстройство & $12(40,0 \pm 9,1 \%)$ & $3(10,0 \pm 5,6 \%)$ \\
\hline
\end{tabular}


Таблица 2.

Распределение пациентов по степени тяжести абдоминального болевого синдрома в группах сравнения

\begin{tabular}{lcc}
\hline \multicolumn{1}{c}{ Степень тяжести боли } & $\begin{array}{c}\text { Основная группа }(\mathrm{n}=30) \\
\text { абс. }(\% \pm \mathrm{m} \%)\end{array}$ & $\begin{array}{c}\text { Группа сравнения }(\mathrm{n}=30) \\
\text { абс. }(\% \pm \mathrm{m} \%)\end{array}$ \\
\hline \hline Легкая & $3(10,0 \pm 5,6 \%)$ & $6(20,0 \pm 7,4 \%)$ \\
\hline Умеренная & $5(16,7 \pm 6,9 \%)$ & $12(40,0 \pm 9,1 \%)$ \\
\hline Сильная & $17(56,7 \pm 9,2 \%)$ & $10(33,3 \pm 8,8 \%)$ \\
\hline Очень сильная & $5(16,7 \pm 6,9 \%)$ & $2(6,7 \pm 4,6 \%)$ \\
\hline
\end{tabular}

При проведении сравнения долей пациентов с различной степенью выраженности симптомов ФГИР между группами сравнения было выявлено, что существует статистически значимое $(\mathrm{p}<0,05)$ различие между размерами долей пациентов с легкой степенью тяжести расстройства. При этом доля данных пациентов в группе сравнения была статистически значимо $(\mathrm{p}<0,05)$ больше. Также было выявлено статистически значимое $(\mathrm{p}<0,05)$ различие между размерами долей пациентов с выраженной степенью тяжести расстройства. При этом доля данных пациентов в основной группе была статистически значимо $(\mathrm{p}<0,05)$ больше. Если можно, лучше одно при этом убрать.

Более высокая степень выраженности клинических симптомов ФГИР среди пациентов основной группы была связана с тем, что в большинстве случаев проявления СРК сочетались с симптомами диспепсии (чувство переполнения после приема пищи, тошнота, раннее насыщение, эпигастральная боль или жжение) $73,3 \pm 8,1 \%$. Среди пациентов группы сравнения лишь у $1 / 3$ больных мы регистрировали симпто-

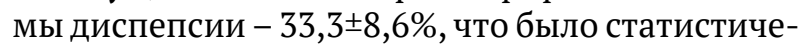
ски значимо $(\mathrm{p}<0,01)$ реже относительно основной группы.

При сравнении степени тяжести абдоминального болевого синдрома как основного клинического проявления СРК с использованием простой описательной шкалы интенсивности боли между группами сравнения установлены значительные отличия (табл. 2.).
Выявлено, что статистически значимо ( $<<0,001)$ наибольшую долю пациентов в основной группе составили больные с сильно выраженным абдоминальным болевым синдромом 56,7 \% (95 \% ДИ 37,8 - 75,5 \%) пациентов. В свою очередь в группе сравнения нами было установлен статистически значимый уровень отличий $(\mathrm{p}<0,05)$ лишь между размерами долей пациентов с умеренным и очень сильным абдоминальным болевым синдромом: 40,0 \% (95 \% ДИ 21,4 - 58,6 \%) и 6,7 \% (95 \% ДИ 2,8 - 16,1 \%) соответственно. У 20,0 \% (95 \% ДИ 4,8 - 35,2 \%) пациентов группы сравнения установлена легкая степень абдоминального болевого синдрома, а у 33,3 \% (95 \% ДИ 15,4 - 51,2 \%) - сильная.

\section{З А К ЛЮ Ч Н ИЕ}

Таким образом, для пациентов с ПИ-СРК, перенесших COVID-19, характерным является более тяжелая клиническая симптоматика заболевания в сравнении с пациентами, страдающими классическим СРК. При этом в большинстве случаев для пациентов, у которых манифестация ФГИР была связана с COVID-19, характерно сочетание функциональных нарушений со стороны кишечника и верхних отделов желудочнокишечного тракта, что проявляется формированием overlap синдрома - сочетанием СРК и функциональной диспепсии. Для пациентов с ПИ-СРК, перенесших COVID-19, также характерна более тяжелая выраженность абдоминального болевого синдрома в сравнении с пациентами с классическим СРК.

\section{А.В. Налетов, Д.В. Каспир, Н.П. Гуз, О.С. Налетова \\ ГОО ВПО «Донецкий национальный медицинский университет имени М. Горького», Донецк \\ ОСОБЕННОСТИ КЛИНИЧЕСКИХ ПРОЯВЛЕНИЙ ПОСТИНФЕКЦИОННОГО СИНДРОМА РАЗДРАЖЕННОГО КИШЕЧНИКА У ПАЦИЕНТОВ, ПЕРЕНЕСШИХ COVID-19}

Одним из наиболее распространенных в популяции функциональных расстройств пищеварительного тракта является синдром раздраженного кишечника. Согласно результатам множества исследований, у
10-50 \% больных с коронавирусной инфекцией отмечаются гастроэнтерологические жалобы. Целью работы было оценить особенности клинического течения постинфекционного синдрома раздраженного 
кишечника у пациентов, перенесших COVID-19. Было обследовано 60 пациентов с синдромом раздраженного кишечника. Для оценки наличия и выраженности основных клинических симптомов функционального гастроинтестинального расстройства у обследованных пациентов нами был использован Опросник «7х7» (7 симптомов за 7 дней). Установлено, что для пациентов с постинфекционным синдромом раздраженного кишечника, перенесших COVID-19, характерным является более тяжелая клиническая сим- птоматика заболевания в сравнении с пациентами с классическим синдромом раздраженного кишечника. При этом в большинстве случаев для пациентов, у которых манифестация расстройства была связана с COVID-19, характерно формирование overlap синдрома -сочетания синдрома раздраженного кишечника и функциональной диспепсии.

Ключевые слова: постинфекционный синдром раздраженного кишечника, COVID-19, опросник «7на7».

\title{
A.V. Nalyotov, D.V. Kaspir, N.P. Guz, O.S. Nalyotova
}

\author{
SEI HPE «M. Gorky Donetsk National Medical University», Donetsk
}

\section{FEATURES OF CLINICAL MANIFESTATIONS OF POST-INFECTIOUS IRRITABLE BOWEL SYNDROME IN PATIENTS WHO HAVE HAD COVID-19}

The irritable bowel syndrome is one of the most common of functional gastrointestinal tract disorders in the population. According to the results of many studies, 10$50 \%$ of patients with coronavirus infection have gastroenterological complaints. The aim of the study was to evaluate the clinical course of post-infectious irritable bowel syndrome in patients who have had COVID-19.60 patients with irritable bowel syndrome were examined. To assess the presence and severity of the main clinical symptoms of functional gastrointestinal disorder we used the $\ll 7 x 7 »$ questionnaire (7 symptoms in 7 days). It was found that patients with post-infectious irritable bowel syndrome who have had COVID-19 are characterized by more severe clinical symptoms of the disease in comparison with patients with classic irritable bowel syndrome. At the same time, in most cases, patients whose manifestation of the disorder was associated with COVID-19 are characterized by the formation of overlap syndrome - a combination of irritable bowel syndrome and functional dyspepsia.

Key words: post-infectious irritable bowel syndrome, COVID-19, questionnaire “7x7».

\section{ЛИТЕРАТУРА}

1. Ивашкин В.Т., Зольникова О.Ю., Охлобыстин А.В. и др. Новая коронавирусная инфекция (COVID-19) и система органов пищеварения. Российскиий журнал гастроэнтерологии, гепатологии, колопроктологии. 2020; 30 (3): 7-13.

2. Wang D., Hu B., Hu C. et al. Clinical characteristics of 138 hospitalized patients with 2019 novel coronavirus-infected pneumonia in Wuhan, China. JAMA. 2020; 323 (11): 1061-1069.

3. Lin L., Jiang X., Zhang Z. et al. Gastrointestinal symptoms of 95 cases with SARS-CoV-2 infection. Gut. 2020; 69 (6): 997-1001.

4. Lacy B.E., Mearin F., Lin Chang et al. Bowel Disorders. Gastroenterology. 2016; 150: 1393-407.

5. Ивашкин В.Т., Шелыгин Ю.А., Баранская Е.К. и др. Клинические рекомендации Российской гастроэнтерологической ассоциации и Ассоциации колопроктологов России по диагностике и лечению синдрома раздраженного кишечника. Российский журнал гастроэнтерологии, гепатологии, колопроктологии. 2017; 27 (5): 76-93.

6. Налетов А.В., Вьюниченко Ю.С., Лянник В.А. Особенности течения синдрома раздраженного кишечника у детей, проживающих в условиях военного конфликта в Донбассе. Университетская клиника. 2018; 1 (26): 5458.

7. Lacy B.E., Moreau J.C. Diarrhea-predominant irritable bowel syndrome: Diagnosis, etiology, and new treatment considerations. J. Am. Assoc. Nurse Pract. 2016; 28 (7): 393-404.

8. Бельмер С.В., Хавкин А.И, Печкуров Д.В. Функциональные расстройства органов пищеварения у детей (в свете Римских критериев IV): методическое пособие для врачей. 2016. 140.

\section{REFERENCES}

1. Ivashkin V.T., Zol'nikova O.Ju., Ohlobystin A.V. i dr. Novaja koronavirusnaja infekcija (COVID-19) i sistema organov pishhevarenija. Rossiiskii zhurnal gastrojenterologii, gepatologii, koloproktologii. 2020; 30 (3): 7-13. (in Russian).

2. Wang D., Hu B., Hu C. et al. Clinical characteristics of 138 hospitalized patients with 2019 novel coronavirus-infected pneumonia in Wuhan, China. JAMA. 2020; 323 (11): 1061-1069.

3. Lin L., Jiang X., Zhang Z. et al. Gastrointestinal symptoms of 95 cases with SARS-CoV-2 infection. Gut. 2020; 69 (6): 997-1001.

4. Lacy B.E., Mearin F., Lin Chang et al. Bowel Disorders. Gastroenterology. 2016; 150: 1393-407.

5. Ivashkin V.T., Shelygin Ju.A., Baranskaja E.K. i dr. Klinicheskie rekomendacii Rossijskoj gastrojenterologicheskoj associacii i Associacii koloproktologov Rossii po diagnostike i lecheniju sindroma razdrazhennogo kishechnika. Rossijskij zhurnal gastrojenterologii, gepatologii, koloproktologii. 2017; 27 (5): 76-93. (in Russian).

6. Naletov A.V., V'junichenko Ju.S., Ljannik V.A. Osobennosti techenija sindroma razdrazhennogo kishechnika u detej, prozhivajushhih $\mathrm{v}$ uslovijah voennogo konflikta $\mathrm{v}$ Donbasse. Universitetskaja klinika. 2018; 1 (26): 54-58. (in Russian).

7. Lacy B.E., Moreau J.C. Diarrhea-predominant irritable bowel syndrome: Diagnosis, etiology, and new treatment considerations. J. Am. Assoc. Nurse Pract. 2016; 28 (7): 393-404.

8. Bel'mer S.V., Havkin A.I, Pechkurov D.V. Funkcional'nye rasstrojstva organov pishhevarenija u detej (v svete Rimskih kriteriev IV): metodicheskoe posobie dlja vrachej. 2016. 140. (in Russian).

9. Ivashkin V.T., Sheptulin A.A., Polujektova E.A.i dr. Vozmozhnost' primenenija Oprosnika «7h7» (sem' simptomov za 
9. Ивашкин В.Т., Шептулин А.А., Полуэктова Е.А. и др. Возможность применения Опросника «7х7» (семь симптомов за семь дней) для оценки динамики симптомов функциональной диспепсии и синдрома раздраженного кишечника. Российский журнал гастроэнтерологии, гепатологии и колопроктологии. 2016. 3: 2433. sem' dnej) dlja ocenki dinamiki simptomov funkcional'noj dispepsii i sindroma razdrazhennogo kishechnika. Rossijskij zhurnal gastrojenterologii, gepatologii i koloproktologii. 2016. 3: 24-33. (in Russian). 